\title{
Chitosan-Pluronic nanoparticles as oral delivery of anticancer gemcitabine: preparation and in vitro study
}

This article was published in the following Dove Press journal:

International Journal of Nanomedicine

5 April 2012

Number of times this article has been viewed

\section{Hosniyeh Hosseinzadeh' \\ Fatemeh Atyabi' \\ Rassoul Dinarvand' \\ Seyed Naser Ostad ${ }^{2}$}

'Nanotechnology Research Center, 2Department of Toxicology and Pharmacology, Faculty of Pharmacy, Tehran University of Medical

Sciences, Tehran, Iran
Correspondence: Fatemeh Atyabi PO Box 14I74, Faculty of Pharmacy, Tehran University of Medical Sciences, Tehran, Iran

Tel +98 2l 66959052

Fax +98 2I 66959052

Email atyabifa@tums.ac.ir
Abstract: Nanoparticles have proven to be an effective delivery system with few side effects for anticancer drugs. In this study, gemcitabine-loaded nanoparticles have been prepared by an ionic gelation method using chitosan and Pluronic ${ }^{\circledR}$ F-127 as a carrier. Prepared nanoparticles were characterized using dynamic light scattering, Fourier transform infrared spectroscopy (FT-IR), differential scanning calorimetry (DSC), scanning electron microscopy, and transmission electron microscopy. Different parameters such as concentration of sodium tripolyphosphate, chitosan, Pluronic, and drug on the properties of the prepared nanoparticles were evaluated. In vitro drug release was studied in phosphate-buffered saline (PBS; $\mathrm{pH}=7.4$ ). The cytotoxicity of the nanoparticles was assayed in the HT-29 colon cancer cell line. The mucoadhesion behavior of the nanoparticles was also studied by mucus glycoprotein assay. The prepared nanoparticles had a spherical shape with positive charge and a mean diameter ranging between 80 to $170 \mathrm{~nm}$. FT-IR and DSC studies found that the drug was dispersed in its amorphous form due to its potent interaction with nanoparticle matrix. Maximum drug encapsulation efficiency was achieved at $0.4 \mathrm{mg} / \mathrm{mL}$ gemcitabine while maximum drug loading was $6 \%$ obtained from $0.6 \mathrm{mg} / \mathrm{mL}$ gemcitabine. An in vitro drug release study at $37^{\circ} \mathrm{C}$ in PBS $(\mathrm{pH}=7.4)$ exhibited a controlled release profile for chitosan-Pluronic ${ }^{\circledR}$ F-127 nanoparticles. A cytotoxicity assay of gemcitabine-loaded nanoparticles showed an increase in the cytotoxicity of gemcitabine embedded in the nanoparticles in comparison with drug alone. The mucoadhesion study results suggest that nanoparticles could be considered as an efficient oral formulation for colon cancer treatment.

Keywords: chitosan, nanoparticles, ionic gelation, gemcitabine, mucoadhesion, oral drug delivery, anticancer

\section{Introduction}

Cancer is one of the major causes of death in many industrialized countries and its incidence is continually increasing. The most common anticancer therapies are tumor removal, radiotherapy, and chemotherapy. Of these methods, chemotherapy is the most effective treatment for tumors. The challenges of anticancer treatment by chemotherapeutic agents include nonselective delivery of cytotoxic drugs to tumor sites that lead to severe side effects due to their effects on normal nontargeted organs and tissues. ${ }^{1-3}$

Nanoscale drug delivery systems have achieved advantages by overcoming the challenges of common cancer treatments. ${ }^{4}$ Nanoparticulate carriers perform as a vehicle protecting the therapeutic agent from the biological milieu and improve cellular uptake and accumulation inside tumor sites. ${ }^{5,6}$ 
Recent anticancer research has focused on polymeric nanoparticles based on chitosan (Chi). Chi is a natural linear polycationic polysaccharide obtained by partial $\mathrm{N}$-deacetylation of chitin. Chi has many advantages as a carrier in nanoparticulate drug delivery systems. It is nontoxic, biocompatible, and biodegradable and has been proven to control the release of drugs, proteins, and peptides. It is soluble in aqueous media, avoids the use of organic solvents, and doesn't require further purification of nanoparticles. ${ }^{7}$ With the presence of free amine groups in its linear structure, Chi has a cationic nature and can interact with various crosslinkers to form nanoparticles. The disadvantages of using chemical crosslinkers such as glutaraldehyde are their toxicity for biological systems, especially if the unreacted crosslinkers are not completely removed from the prepared nanoparticles. To resolve the toxic effects of using chemical crosslinkers, Chi can be ionically crosslinked with multivalent anions like tripolyphosphate. This process, known as ionic gelation, has some advantages since it is a mild process resulting in nanoparticles with sizes less than $200 \mathrm{~nm}$ and has been proven to encapsulate different biological and active compounds. ${ }^{8-10}$

The positive charge of Chi caused by the primary amino groups in its structure is responsible for its mucoadhesive properties and therefore prolonging the residual time at the absorption site. Chi nanoparticles are expected to be appropriate carriers for oral absorption of drugs. ${ }^{11,12}$

Pluronic ${ }^{\circledR}$ F-127 (PF) is a hydrophilic nontoxic copolymer widely used as a pharmaceutical excipient for its stabilizing properties and capability to increase the solubility of drugs. ${ }^{13}$

Pluronic ${ }^{\circledR}$ F-127 is an A-B-A-type triblock copolymer consisting of polyoxyethylene (PEO) units (A) and polyoxypropylene (PPO) units (B) with a thermoreversible gelation property. ${ }^{14} \mathrm{With}$ the increase in the temperature of $\mathrm{PF}$ aqueous solution, the PPO block tends to dehydrate and form a core with an outer shell of hydrated PEO chains that aggregate into spherical micelles. ${ }^{15-18}$ The micellar structure of this copolymer in an aqueous environment can be used for incorporation of hydrophilic and hydrophobic drugs ${ }^{19}$ and prolongs drug release..$^{13,20,21}$

Gemcitabine (GC; $2^{\prime}, 2^{\prime}$ difluorodeoxycytidine) is a pyrimidine antimetabolite that is broadly used for management of a variety of solid tumors including colon, pancreatic, lung, breast, ovarian, and bladder. After energy-dependent uptake of GC into cells, it is phosphorylated into its active metabolite, gemcitabine triphosphate and diphosphate mainly via deoxycytidine kinase..$^{22,23}$

The active metabolites are incorporated into a replicating DNA strand, blocking DNA polymerase activity and inhibiting DNA synthesis. ${ }^{24}$ Since GC is a water-soluble low-molecular-weight anticancer drug, it requires nucleoside transporters localized on the membrane to enter the cells..$^{25,26}$ Different approaches (using different drug delivery systems) were performed to improve its delivery to tumor sites. Formulations such as prodrug conjugates, liposomes, polymeric nanoparticles, and nanoconjugates were designed in order to protect GC from rapid metabolization, overcome drug resistance, target drug delivery, and improve efficacy via different routes of administration. ${ }^{27-32}$

As most anticancer drugs are available in injectable forms, improving the feasibility of oral administration of an anticancer drug would offer the advantages of oral routes especially for drugs such as GC that have low systemic exposure following oral administration. The drug-carrying combination of Chi and PF offer promising combinations by modifying the controlled drug release profile using PF with protection and transfection-enhancing effects using Chi.

To our knowledge, no study was done on GC nanoparticles prepared by ionic gelation using the combination of Chi and PF as carriers. By using Chi as a mucoadhesive polymer, we expect this system to be more cytotoxic than pristine drug by delivering the nanoparticles to HT-29 colon carcinoma cell line.

\section{Materials}

Gemcitabine was obtained from Cipla (Mumbai, India). Medium-molecular-weight Chi with a degree of deacetylation of about $89 \%$ was purchased from Primex (Karmoy, Norway). Sodium nitrite $\left(\mathrm{NaNO}_{2}\right), \mathrm{PF}$, sodium tripolyphosphate (TPP), hydrochloric acid, glacial acetic acid, sodium hydroxide $(\mathrm{NaOH})$, mucin, basic fuchsin, periodic acid, sodium metabisulfite, ethyl cellulose, Carbomer 940, and potassium hydrogen phosphate were all purchased from Merck (Darmstadt, Germany). 3-(4,5-dimethylthiazol-2-yl)2,5-diphenyltetrazolium bromide) (MTT) was purchased from Sigma-Aldrich (St Louis, MO). The HT-29 cell line was obtained from Pasteur Institute (Tehran, Iran). All chemicals were of analytical grade.

\section{Methods}

\section{Preparation of nanoparticles}

\section{Preparation of low-molecular-weight chitosan}

To obtain a low-molecular-weight Chi, medium molecular weight Chi was depolymerized according to the method described by Moghaddam et al. ${ }^{33}$ Briefly, Chi (400 kDa) was dissolved in acetic acid $(6 \% \mathrm{v} / \mathrm{v})$ to obtain a solution of $2 \% \mathrm{v} / \mathrm{v}$ Chi in acetic acid. Low-molecular-weight Chi was 
obtained after addition of $10 \mathrm{~mL}$ of $\mathrm{NaNO}_{2}(7.0 \mathrm{mg} / \mathrm{mL})$ to the dissolved Chi at room temperature under magnetic stirring. After 1 hour, the depolymerized Chi was precipitated by raising the $\mathrm{pH}$ to 9.0 by adding $\mathrm{NaOH}(4 \mathrm{~N})$. The whiteyellowish solid was filtrated, washed with acetone three times, and dissolved in a minimum volume of $0.1 \mathrm{~N}$ acetic acid. Purification was carried out by subsequent dialysis against purified water (Sigma dialysis tubes, molecular weight cutoff $12 \mathrm{kDa}$; Sigma Aldrich). The dialyzed product was lyophilized using a freeze dryer (Alpha 2-4 LD plus; Christ, Osterode am Harz, Germany). The yellowish lyophilized product was then stored at $4^{\circ} \mathrm{C}$ until further use. The average molecular weight of the prepared Chi was determined by gel permeation chromatography (Agilent Technologies, Santa Clara, CA) using plulane according to the method used in our lab by Akhlaghi et al. ${ }^{34}$

The preparation of Chi nanoparticles was achieved via the ionic gelation method reported by Calvo et al. ${ }^{8} \mathrm{~A}$ Chi solution $(0.1 \% \mathrm{w} / \mathrm{v})$ was obtained by dissolving low-molecular-weight Chi in $1 \% \mathrm{v} / \mathrm{v}$ acetic acid. Chi nanoparticles were prepared spontaneously upon addition of various concentrations of TPP $(0.015 \%, 0.02 \%, 0.025 \%$, and $0.03 \% \mathrm{w} / \mathrm{v})$ to Chi solution under gentle magnetic stirring at room temperature for 1 hour. In all cases, the volume ratio of Chi:TPP solution was 2:1. Finally the opaque suspension was assigned as nanoparticles. ${ }^{8}$ This zone was obtained at TPP solution ranging $0.015 \%-0.025 \% \mathrm{w} / \mathrm{w}$. PF was incorporated into nanoparticles by adding $0.025 \% \mathrm{w} / \mathrm{v}$ TPP solution to Chi aqueous solution containing different concentrations of PF $(10 \%, 15 \%, 20 \% \mathrm{w} / \mathrm{w})$.

For preparation of GC-loaded nanoparticles, various concentrations of GC $(0.2,0.4,0.6,0.8$ and $1.0 \mathrm{mg} / \mathrm{mL})$ in $0.025 \% \mathrm{w} / \mathrm{w}$ TPP solution were prepared. Nanoparticles were formed by adding this solution into Chi in an acetic acid solution.

The nanoparticles were separated from the aqueous medium by Amicon Ultra-15 centrifugal filters (Millipore, Billerica, MA) at $5000 \mathrm{~g}$, at room temperature until removal of the nanoparticles from the preparation media (20 minutes). The nanoparticles were removed from the filter and suspended in $2 \mathrm{~mL}$ of deionized water. This suspension was freeze-dried and stored at $4^{\circ} \mathrm{C}$ until use.

\section{Characterization of Chi nanoparticles}

\section{Size measurements and determination} of zeta potential

The mean diameter and size distribution of the nanoparticles were measured by dynamic light scattering using Zetasizer
(Nano-ZS; Malvern Instruments, Malvern, UK). All measurements were performed with a wavelength of $633 \mathrm{~nm}$ at $25^{\circ} \mathrm{C}$ with an angle detection of $90^{\circ} \mathrm{C}$. The zeta potential of freshly prepared nanoparticles was determined by laser Doppler electrophoresis using Zetasizer (Malvern Instruments). Each sample measurement was repeated three times.

Evaluation of drug encapsulation and loading capacity The loading capacity (LC) and encapsulation efficiency (EE) were measured indirectly by measuring the amount of remaining drug in the medium collected at the bottom of the falcon using UV spectrometry (CE7500; Cecil, Cambridge, UK) at $274 \mathrm{~nm}$. Each sample was measured in triplicate.

The following equations were used to evaluate the LC and EE of the nanoparticles: ${ }^{36}$

$$
\begin{aligned}
& \text { EE }(\%)=\frac{\text { Total GC }- \text { Free GC }}{\text { Total GC }} \times 100 \\
& \text { LC }(\%)=\frac{\text { Total GC }- \text { Free GC }}{\text { Nanoparticle weight }} \times 100
\end{aligned}
$$

\section{Scanning electron microscopy}

The surface morphology of the freshly prepared nanoparticles was observed using a scanning electron microscope (XL 30; Philips, Eindhoven, The Netherlands). Nanoparticles were dried on an aluminum disk at room temperature. The fixed nanoparticles were coated with gold using a sputter coater (SCD 005; Bal-Tec, Balzers, Liechtenstein).

\section{Transmission electron microscopy}

Transmission electron microscopy (TEM; CEM 902A; Carl Zeiss, Oberkochen, Germany) was used to examine and compare the topography of the nanoparticles. Freeze-dried nanoparticles were suspended in deionized water before observation.

\section{Fourier transform infrared spectroscopy}

Fourier transform infrared (FT-IR) spectra were analyzed using a Nicolet FT-IR Spectrometer (Magna IR 550; Madison, WI) at $4 \mathrm{~cm}^{-1}$ resolution. The freeze-dried nanoparticles were mixed with $\mathrm{KBr}$ and pressed to a plate to investigate the chemical reactions between the drug and nanoparticle matrix.

\section{Differential scanning calorimetry}

Differential scanning calorimetry (Mettler Toledo, DSC 823 ${ }^{\mathrm{e}}$, Greifensee, Switzerland) was used to determine the physical 
status of GC in nanoparticles. An appropriate amount of samples $(5-10 \mathrm{mg})$ were sealed in aluminum pans and scanned in a temperature range of 0 to $550^{\circ} \mathrm{C}$ with a heating rate of $20^{\circ} \mathrm{C} /$ minute. Inert atmosphere was maintained by purging nitrogen with a flow rate of $360 \mathrm{~cm}^{3} /$ minute.

\section{In vitro drug release}

Four milligrams of nanoparticles were dispersed in a freshly prepared phosphate-buffered saline (PBS; $\mathrm{pH}=7.4$ ) as a release medium in a dialysis membrane sac (mw cut-off $12 \mathrm{kDa}$; Sigma Aldrich). The enclosed dialysis sac was immersed in a beaker containing $50 \mathrm{~mL}$ of the release medium. The beaker was placed in a shaking incubator at $37^{\circ} \mathrm{C}$ under mild agitation (90-100 rpm). For each sample, $5 \mathrm{~mL}$ of the release medium was withdrawn at predetermined time intervals and replaced by the same medium at the same condition. The samples were analyzed for drug content by ultraviolet spectrometry as described above.

\section{Mucoadhesion studies}

Mucoadhesion studies of Chi and Chi-PF nanoparticles were performed by mucus glycoprotein assay. Periodic acid/Schiff colorimetric method was used for determining the amount of free mucin to estimate the amount of adsorbed mucins on the nanoparticles. Schiff reagent was prepared by adding $1 \mathrm{~g}$ basic fuchsin to $100 \mathrm{~mL}$ water. Twenty microliters of $\mathrm{HCl}$ (2M) was added to the prepared fuchsin solution following addition of $0.1 \mathrm{~g}$ sodium metabisulfite to the Schiff reagent, and the final solution was incubated at $37^{\circ} \mathrm{C}$ until alteration of the color from brown to orange. Periodic acid reagent was freshly prepared by adding $10 \mu \mathrm{L}$ of $50 \% \mathrm{v} / \mathrm{v}$ periodic acid solution to $7.0 \mathrm{~mL}$ of $7 \% \mathrm{v} / \mathrm{v}$ acetic acid solution. Standard solutions of mucin $(0.25,0.5,0.75$, and $1.0 \mathrm{mg}$ per $2.0 \mathrm{~mL})$ were used to prepare standard calibration curves. After adding $0.20 \mathrm{~mL}$ periodic acid reagent to the samples, the samples were incubated at $37^{\circ} \mathrm{C}$ in a water bath for 2 hours. Then, at room temperature, $0.20 \mathrm{~mL}$ Schiff reagent was added to the samples. After 30 minutes the absorbance of the solution was recorded at $556 \mathrm{~nm}$ in an ultraviolet spectrophotometer. A standard calibration curve was plotted to calculate the mucin content adsorbed to nanoparticles. ${ }^{37}$

To determine the mucoadhesion of the nanoparticles, $10 \mathrm{mg}$ of the nanoparticles (Chi and Chi-PF PF $=20 \% \mathrm{w} / \mathrm{w}$ ) was dispersed in $6 \mathrm{~mL}$ of the mucin solution $(0.5 \mathrm{mg} / \mathrm{mL})$. The suspensions were incubated for 1 hour at $37^{\circ} \mathrm{C}$ while being shaken. In order to analyze unadsorbed free mucin, the suspensions were then centrifuged (12,000 rpm for 5 minutes), and the supernatants analyzed by spectrophotometer at the visible wavelength of $555 \mathrm{~nm}$. The rest of the procedure was the same as for the standard solutions. Ethylcellulose was used as negative control, and Carbomer 940 was used as positive control for comparison purposes. Both controls were assessed with the same procedure as described previously.

\section{Cytotoxicity test}

To assess the viability of HT-29 colon carcinoma against GC, blank nanoparticles (Chi, Chi-PF), and drug-loaded nanoparticles were tested using MTT assay. The HT-29 cell line was seeded in 24-well plates at a density of 150,000 viable cells per well and incubated for 24 hours to allow cell attachment. The cells were then incubated for another 24 hours with blank nanoparticles (Chi, Chi-PF) and GC-loaded nanoparticles (GC-Chi, GC-Chi-PF). Cells were then washed in PBS, and $200 \mu \mathrm{L}$ of MTT solution $(5 \mathrm{mg} / \mathrm{mL})$ was added to each well. The plates were incubated for an additional 4 hours, and then the medium was discarded. Dimethyl sulfoxide $(600 \mu \mathrm{L})$ was added to each well, and the solution was vigorously mixed to dissolve tetrazolium dye. Then $100 \mu \mathrm{L}$ of the dissolved dye from each of the wells was transferred into 96-well plates. The absorbance of each well was measured by enzyme-linked immunosorbent assay reader (Anthous 2020; AnthosLabtec Instruments, Salzburg, Austria) at a test wavelength of $570 \mathrm{~nm}$ against a standard reference solution at $690 \mathrm{~nm}$. Nanoparticles were sterilized by membrane filtration $(0.2$ $\mu \mathrm{m})$ in a laminar hood before MTT assay.

\section{Statistical analysis}

Results are shown as mean \pm standard deviation. Statistical data analyses were performed using statistical software program (SPSS 16; SPSS Inc, Chicago, IL). Comparison between data was done using one-way analysis of variance with a $P$-value $<0.05$ as the minimal level of significance.

\section{Results and discussion Mean diameter, size distribution, and zeta potential of nanoparticles}

To obtain nanoparticles with sizes below $200 \mathrm{~nm}$, nanoparticles were prepared utilizing low-molecular-weight Chi $(16-20 \mathrm{kDa}) .{ }^{37}$ The nanoparticles were prepared by ionic gelation upon addition of TPP to Chi in acetic acid under gentle magnetic stirring at room temperature. TPP has five negative ionic charge points that interact with the positive amino groups of Chi in acetic acid solution. Different parameters influence the characters of the nanoparticles. These include $\mathrm{pH},{ }^{38,39}$ molecular weight of $\mathrm{Chi},{ }^{40} \mathrm{Chi}$ and 
TPP concentration, addition of a second polymer/copolymer, and addition of an active compound. , $^{8,35}$

Among these parameters, Chi and TPP are the most important factors that control the size of the prepared nanoparticles. Chi and TPP can form nanoparticles in specific moderate concentrations. ${ }^{8}$ Nanoparticles with smaller size have valuable characteristics such as improved drug delivery, longer circulation in blood, and lower toxicity. ${ }^{5,35}$

Table 1 shows the effect of increasing TPP concentration on particle size. It was observed that application of TPP with higher concentration can significantly increase the size of the particles $(P<0.002)$. This could be due to the increase in the amount of anionic groups in the preparation medium, which causes more electrostatic interaction with positive amino sites on Chi, reduction of the positive surface charge, and increments in nanoparticles size $(P<0.003)$. Zeta potential influences the stability of the nanoparticles through electrostatic repulsion. ${ }^{38,41} \mathrm{TPP}$ concentrations higher than $0.03 \% \mathrm{w} / \mathrm{v}$ form aggregated solutions, which are not confirmed as nanoparticles according to the report by Calvo et al. ${ }^{8}$

Table 2 demonstrates that the size of Chi nanoparticles increases as Chi concentration is increased. In all cases, TPP concentration was kept constant $(0.025 \% \mathrm{w} / \mathrm{v})$. The increased viscosity of higher Chi concentrations prevents effective ionic interaction between TPP and Chi solution, which increases nanoparticle $\operatorname{size}^{42}$ with increase in zeta potential $(P<0.007)$.

We prepared nanoparticles containing PF with the intention to make nanoparticles with a controlled release profile. ${ }^{13}$ The addition of PF results in decreased size $(P<0.036)$, which can be attributed to the formation of a rigid gel leading to lesser water uptake. Slighter swelling causes a reduction in the mean diameter of the particles in aqueous medium (Table 3). ${ }^{43}$ Since there is no significant change in zeta potential by increasing concentrations of PF $(P<0.586)$, it can be concluded that PF is mostly incorporated inside the nanoparticle matrix. This may be due to the PF concentration. In the

Table I Effect of TPP concentration on mean diameter and zeta potential of Chi nanoparticles

\begin{tabular}{lclr}
\hline $\begin{array}{l}\text { TPP } \\
\text { concentration } \\
(\% w / v)\end{array}$ & $\begin{array}{l}\text { Mean } \\
\text { diameter } \\
(\mathbf{n m})\end{array}$ & $\begin{array}{l}\text { Polydispersity } \\
\text { index }\end{array}$ & \multicolumn{1}{l}{$\begin{array}{l}\text { Zeta } \\
\text { potential } \\
(\mathbf{m v})\end{array}$} \\
\hline 0.015 & $80 \pm 5.65$ & $0.230 \pm 0.36$ & $35.2 \pm 0.62$ \\
0.02 & $109 \pm 2.82$ & $0.208 \pm 0.50$ & $29.85 \pm 1.49$ \\
0.025 & $113 \pm 5.29$ & $0.186 \pm 0.027$ & $26.2 \pm 1.76$ \\
0.03 & $166.6 \pm 8.08$ & $0.194 \pm 0.018$ & $18.13 \pm 0.55$ \\
\hline
\end{tabular}

Note: $\mathrm{Chi}=0.1 \% \mathrm{w} / \mathrm{v}$.

Abbreviations: Chi, chitosan; TPP, sodium tripolyphosphate.
Table 2 Effect of Chi concentration on mean diameter and zeta potential of Chi nanoparticles

\begin{tabular}{llll}
\hline $\begin{array}{l}\text { Chi } \\
\text { concentration } \\
(\% w / v)\end{array}$ & $\begin{array}{l}\text { Mean } \\
\text { diameter } \\
(\mathbf{n m})\end{array}$ & $\begin{array}{l}\text { Polydispersity } \\
\text { index }\end{array}$ & $\begin{array}{l}\text { Zeta } \\
\text { potential } \\
(\mathbf{m v})\end{array}$ \\
\hline 0.2 & $148 \pm 1.67$ & $0.261 \pm 0.026$ & $35.8 \pm 0.54$ \\
0.4 & $242 \pm 2.56$ & $0.358 \pm 0.047$ & $38.2 \pm 1.25$ \\
0.6 & $256 \pm 1.45$ & $0.312 \pm 0.029$ & $44.1 \pm 2.31$ \\
0.8 & $328 \pm 3.32$ & $0.379 \pm 0.034$ & $45.6 \pm 0.76$ \\
1 & $452 \pm 5.43$ & $0.433 \pm 0.053$ & $46.9 \pm 2.46$ \\
1.2 & $584 \pm 3.47$ & $0.486 \pm 0.041$ & $49.8 \pm 1.78$ \\
\hline
\end{tabular}

Note: TPP $=0.025 \% \mathrm{w} / \mathrm{v}$.

Abbreviations: Chi, chitosan; TPP, sodium tripolyphosphate.

concentrations utilized in this study, conformational change in the copolymer molecule occurs and gives rise to a closepacked monomolecular unit with the poly(oxypropylene) hydrophobic core surrounded by poly(oxyethylene) units or the formation of multimolecular aggregates that are trapped inside the nanoparticle matrix. ${ }^{44}$ In this case, PF does not seem to be attached to the surface of the nanoparticles, thus it does not influence the surface charge of the particles.

GC-loaded nanoparticles were prepared upon addition of GC in $0.025 \% \mathrm{w} / \mathrm{v}$ TPP into $0.1 \% \mathrm{w} / \mathrm{v}$ Chi or Chi-PF in acetic acid solution. To determine the effect of GC concentration on particle size, various concentrations of $\mathrm{GC}$ in $0.025 \%$ w/v TPP solution were applied. Table 4 shows that generally addition of GC increases the size of Chi nanoparticles, but does not significantly affect their zeta potential $(P<0.865)$. The effect of GC concentration on particle size is more significant when the concentration rises from 0.4 to $0.6 \mathrm{mg}$ / $\mathrm{mL}(P<0.002)$.

In general, GC-loaded Chi-PF nanoparticles size did not grow significantly at concentrations up to $0.4 \mathrm{mg} /$ $\mathrm{mL}(P<0.586)$, but there was a change in size when the concentration of GC was increased from 0.4 to $0.6 \mathrm{mg} / \mathrm{mL}$ $(P<0.021)$, and the size remains constant at concentrations above $0.6 \mathrm{mg} / \mathrm{mL}$ of GC in TPP solution (Table 5). GC is a low-molecular-weight anticancer drug. Therefore it may not be possible to severely increase the GC particle diameter until

Table 3 Effect of PF concentration on mean diameter and zeta potential of Chi nanoparticles

\begin{tabular}{llll}
\hline PF $(\% w / w)$ & Mean diameter & Pdl & Zeta potential \\
\hline 10 & $128 \pm 2.64$ & $0.165 \pm 0.002$ & $25.46 \pm 0.75$ \\
15 & $117 \pm 3.0$ & $0.221 \pm 0.068$ & $23.86 \pm 1.8$ \\
20 & $107 \pm 3.29$ & $0.148 \pm 0.007$ & $23.46 \pm 1.2$ \\
\hline
\end{tabular}

Notes: $\mathrm{Chi}=0.1 \% \mathrm{w} / \mathrm{v}, \mathrm{TPP}=0.025 \% \mathrm{w} / \mathrm{v}$.

Abbreviations: Chi, chitosan; Pdl, Polydispersity Index; PF, Pluronic F ${ }^{\circledR}$ 127; TPP, sodium tripolyphosphate. 
Table 4 Characterization of Chi nanoparticles by varying GC concentration

\begin{tabular}{lclrll}
\hline $\mathbf{G C}(\mathbf{m g} / \mathbf{m L})$ & Mean diameter $(\mathbf{n m})$ & PdI & Zeta potential $(\mathbf{m v})$ & EE $(\%)$ & \multicolumn{1}{l}{ LC $(\%)$} \\
\hline- & $113 \pm 5.29$ & $0.186 \pm 0.027$ & $26.2 \pm 1.76$ & - & - \\
0.2 & $123.5 \pm 3.53$ & $0.143 \pm 0.026$ & $25.9 \pm 0.71$ & $63.68 \pm 0.21$ & $3.94 \pm 0.40$ \\
0.4 & $130.66 \pm 3.21$ & $0.211 \pm 0.031$ & $25.46 \pm 1.15$ & $71.07 \pm 0.39$ & $5.26 \pm 0.42$ \\
0.6 & $154 \pm 4.58$ & $0.157 \pm 0.066$ & $24.75 \pm 0.77$ & $67.25 \pm 1.89$ & $6.01 \pm 0.28$ \\
0.8 & $157 \pm 6.2$ & $0.199 \pm 0.043$ & $24.4 \pm 1.69$ & $65.44 \pm 1.13$ & $5.48 \pm 0.47$ \\
$I$ & $156.33 \pm 4.04$ & $0.310 \pm 0.056$ & $24.06 \pm 2.05$ & $63.79 \pm 2.88$ & $5.56 \pm 0.53$ \\
\hline
\end{tabular}

Notes: $\mathrm{Chi}=0.1 \% \mathrm{w} / \mathrm{v}, \mathrm{TPP}=0.025 \%$.

Abbreviations: Chi, chitosan; EE, encapsulation efficiency; GC, gemcitabine; LC, loading capacity; PdI, Polydispersity Index; PF, Pluronic F ${ }^{\circledR}$ 27; TPP, sodium tripolyphosphate.

it reaches its maximum capacity inside the nanoparticles. GC concentration did not influence the zeta potential of the prepared nanoparticles $(P<0.944)$.

\section{Nanoparticle encapsulation efficiency and loading capacity}

The effect of different concentrations of GC on the EE and LC of Chi nanoparticles was determined. The observed results did not show a significant change in EE of nanoparticles $(P<0.1)$, but maximum EE $(71 \%)$ was achieved at $0.4 \mathrm{mg} / \mathrm{mL}$ of GC concentration (Table 4). While maximum LC was observed at $0.6 \mathrm{mg} / \mathrm{mL}$ of GC in TPP solution, the increase in drug concentration from $0.4 \mathrm{mg} / \mathrm{mL}$ to $1 \mathrm{mg} / \mathrm{mL}$ did not significantly affect the LC $(P<0.228)$.

To determine the effect of $\mathrm{PF}$ incorporation on the $\mathrm{EE}$ and LC of the nanoparticles, $0.6 \mathrm{mg} / \mathrm{mL}$ of GC in TPP was added to different amounts of PF $(10 \%, 15 \%, 20 \% \mathrm{w} / \mathrm{w})$ in Chi in acetic acid solution. Augmentation of PF reduced EE $(P<0.024)$, but did not significantly influence the LC of the nanoparticles $(P<0.126)$ (Table 6). As analysis of FT-IR spectrum shows, GC interacts via a hydrogen bond and electrostatic interaction with the free positive amine groups available inside the nanoparticles. PF competes with GC in interacting with Chi by occupying the functional sites

Table 5 Effect of GC concentration on the mean diameter and zeta potential of Chi-PF nanoparticles

\begin{tabular}{lllr}
\hline $\begin{array}{l}\text { GC } \\
\text { concentration } \\
(\mathbf{m g} / \mathbf{m L})\end{array}$ & $\begin{array}{l}\text { Mean } \\
\text { diameter } \\
(\mathbf{n m})\end{array}$ & PdI & \multicolumn{1}{l}{$\begin{array}{l}\text { Zeta } \\
\text { potential } \\
(\mathbf{m v})\end{array}$} \\
\hline- & $107 \pm 3.29$ & $0.210 \pm 0.011$ & $23.50 \pm 1.32$ \\
0.2 & $102 \pm 1.92$ & $0.167 \pm 0.028$ & $25.42 \pm 0.87$ \\
0.4 & $98 \pm 1.43$ & $0.193 \pm 0.015$ & $24.9 \pm 2.10$ \\
0.6 & $115 \pm 2.12$ & $0.167 \pm 0.023$ & $25.3 \pm 0.65$ \\
0.8 & $109 \pm 3.10$ & $0.22 \pm 0.031$ & $24.7 \pm 1.98$ \\
\hline & $111 \pm 1.64$ & $0.154 \pm 0.018$ & $25.12 \pm 1.57$ \\
\hline
\end{tabular}

Note: $\mathrm{PF}=20 \% \mathrm{w} / \mathrm{w}$.

Abbreviations: Chi, chitosan; GC, gemcitabine; Pdl, Polydispersity Index; PF, Pluronic F ${ }^{\circledR} / 27$. of the Chi. The small molecular structure of GC cannot overcome the hindrance effect of PF, therefore incorporation of PF leads to reduced EE and therefore lower LC in comparison with Chi nanoparticles. The same results were reported by Wu et al when poly(ethylene glycol) (PEG) was added prior to gelation of Chi with TPP. Addition of PEG led to a lower encapsulation of ammonium glycyrrhizinate into the nanoparticles. ${ }^{45}$

\section{Morphology of nanoparticles}

SEM micrographs (Figure 1) of Chi and Chi-PF nanoparticles loaded with GC, show that particles are spherical and uniform. TEM images of the nanoparticles also confirms the spherical shape. In Chi-PF nanoparticles, darker spots were observed inside the spherical matrix of the nanoparticles (Figure 2B), while they are not seen in Chi nanoparticles (Figure 2A). As both NP formulations prepared for TEM imaging were loaded with GC, thus, these darker spots are responsible for the contrast observed between Chi and PF. This micrograph proves the incorporation of $\mathrm{PF}$ inside the nanoparticle matrix and disproves the attachment of PF on the nanoparticle surface.

\section{Interaction between GC}

\section{and nanoparticle matrix}

FT-IR spectrometry was used to find out the nature of interaction between GC, Chi, or TPP. Various physicochemical interactions between GC, Chi, PF, and TPP can alter the

Table 6 Encapsulation efficiency and loading capacity of Chi-PF with different PF concentrations

\begin{tabular}{lll}
\hline PF $(\% w / w)$ & EE (\%) & LC (\%) \\
\hline 10 & $68.47 \pm 1.97$ & $5.43 \pm 0.12$ \\
15 & $63.67 \pm 0.78$ & $4.85 \pm 0.35$ \\
20 & $56.22 \pm 1.56$ & $4.33 \pm 0.26$ \\
\hline
\end{tabular}

Notes: $\mathrm{GC}=0.6 \mathrm{mg} / \mathrm{mL}, \mathrm{Chi}=0.1 \% \mathrm{w} / \mathrm{v}, \mathrm{TPP}=0.025 \% \mathrm{w} / \mathrm{v}$.

Abbreviations: Chi, chitosan; EE, encapsulation efficiency; GC, gemcitabine; LC, loading capacity; PF, Pluronic F⿶127. 


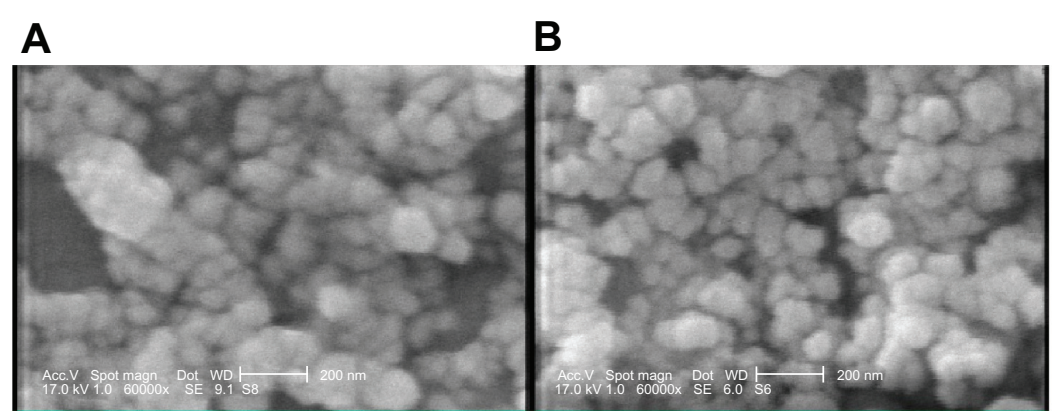

Figure I SEM micrographs of GC-loaded (A) Chi nanoparticles (B) Chi-PF nanoparticles.

Abbreviations: Chi, chitosan; GC, gemcitabine; PF, Pluronic $\mathrm{F}^{\circledR}$ |27; SEM, scanning electron microscopy.

absorption peaks or broaden them. Nanoparticles are formed in an aqueous acidic medium, which leads to a positive charge for Chi due to the existence of a primary amino group on its molecular structure that can form an electrostatic interaction with TPP. In addition, by the existence of fluorine, hydrogen, oxygen, and nitrogen atoms in GC molecular structure (Figure 3), we expect hydrogen bonding between GC and nanoparticle matrix. In the spectra of pure Chi in Figure $4 \mathrm{C}$, the vibrational band at $1638.3 \mathrm{~cm}^{-1}$ represents the primary amino group in Chi, while the stretching bands at $1073.9 \mathrm{~cm}^{-1}, 1429.7 \mathrm{~cm}^{-1}$, and $3442.4 \mathrm{~cm}^{-1}$ are due to the $\mathrm{C}-\mathrm{O}, \mathrm{C}-\mathrm{H}$, and hydroxyl groups present in $\mathrm{Chi}$, respectively. Two peaks at $2918.7 \mathrm{~cm}^{-1}$ and $2852.8 \mathrm{~cm}^{-1}$ show the stretching band of methylene in Chi structure. For blank Chi nanoparticles (Figure 4B), the amino band is shifted to $1550.3 \mathrm{~cm}^{-1}$, which indicates the ionic interaction between TPP and $\mathrm{NH}_{2}$ of Chi. The broad band with a maximum at $3370.8 \mathrm{~cm}^{-1}$ represents hydrogenic bonds between hydroxyl groups in Chi with TPP. These interactions lead to a decrease in Chi solubility and nanoparticle formation. In the case of GC (Figure 4D), there is a characteristic peak at $1743.7 \mathrm{~cm}^{-1}$, which corresponds to the ureido group in GC molecular structure (Figure 3D). A peak at $3440.8 \mathrm{~cm}^{-1}$ is due to the overlapped hydroxyl and amino bands in this area. When GC was loaded in Chi nanoparticles (Figure 4A), a small band at $1735.7 \mathrm{~cm}^{-1}$ appeared. On the other side, the free amino band at $1638.3 \mathrm{~cm}^{-1}$ vanished and the peak at $1436.6 \mathrm{~cm}^{-1}$ broadened. Therefore a strong interaction between GC and amino groups of Chi has occurred. The broad band at $3000-3500 \mathrm{~cm}^{-1}$ indicates the strong hydrogen bond between drug and nanoparticle matrix. For PF (Figure 5B), a strong stretching band at $2870 \mathrm{~cm}^{-1}$ represents the stretching vibrational band of methylene group. As in blank Chi-PF nanoparticles (Figure 5A), this peak remains and confirms the incorporation of PF in Chi-PF nanoparticles. For GCloaded Chi-PF nanoparticles (Figure 5C), the ureido peak exists, which authenticates the encapsulation of GC in the nanoparticle matrix. In GC-loaded Chi-PF nanoparticles, the primary amino peak of Chi at $1560 \mathrm{~cm}^{-1}$ in blank nanoparticles was shifted to $1443.1 \mathrm{~cm}^{-1}$. The same peak exists in GC-loaded Chi nanoparticles (Figure 4A). This may prove the potent interaction of the amino group of Chi with the drug. One possible mechanism can be the ionic interaction between $\mathrm{GC}$ and $\mathrm{NH}_{2}$ of $\mathrm{Chi}$ in addition to the hydrogen bond. Electron resonance between $\mathrm{NH}_{2}$ and the $\mathrm{C}=\mathrm{O}$ functional group inside the pyrimidine structure of GC in TPP solution $(\mathrm{pH}=9)$, leads to a negative charge on carbonyl group in the molecular structure of GC. This negative charge interacts
A

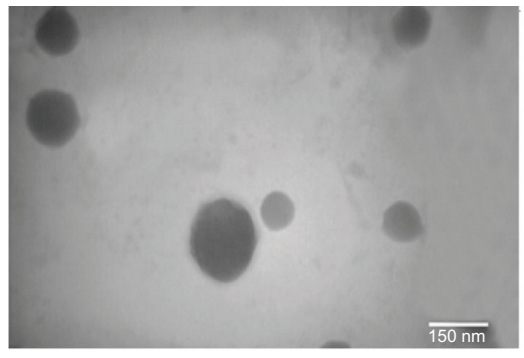

B

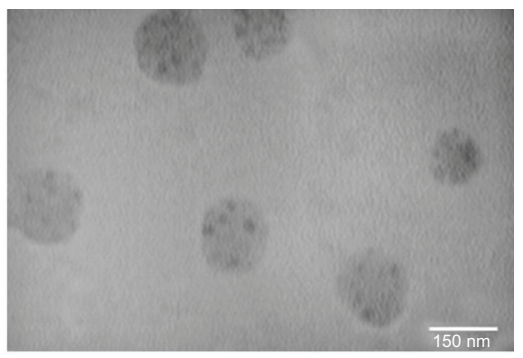

Figure 2 TEM micrographs of GC-loaded (A) Chi nanoparticles (B) Chi-PF nanoparticles.

Abbreviations: Chi, chitosan; GC, gemcitabine; PF, Pluronic $\mathrm{F}^{\circledR}$ /27; TEM, transmission electron microscopy. 
A

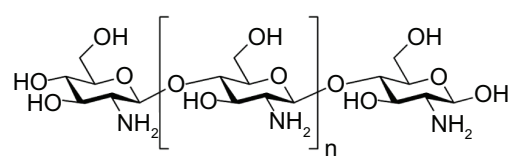

B

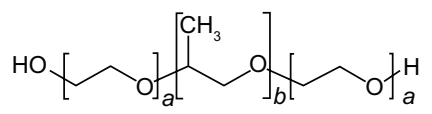

C

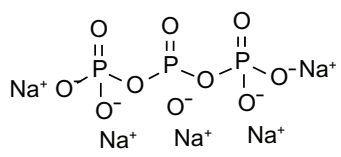

D

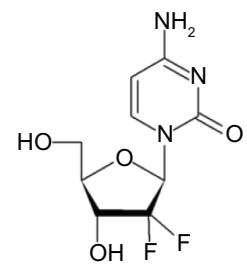

Figure 3 Schemes for (A) chitosan, (B) Pluronic ${ }^{\circledast}$, (C) sodium tripolyphosphate, and (D) gemcitabine.

with the positive amino group of $\mathrm{Chi}$ in acetic acid solution when mixed together to form nanoparticles.

\section{Physical status of GC in nanoparticles}

To confirm the physical state of the drug in nanoparticles, drug alone, blank nanoparticles of Chi and Chi-PF, and drug-loaded nanoparticles of the mentioned formulations were examined with DSC. As shown in Figure 6D, the melting point of drug alone is observed with a sharp peak at $291.61{ }^{\circ} \mathrm{C}$. In blank Chi nanoparticles (Figure 6B), there was a broad endothermic peak at $130.22^{\circ} \mathrm{C}$ and an exothermic peak at $298.82^{\circ} \mathrm{C}$. In blank Chi-PF nanoparticles (Figure 6F), an endothermic peak at $57.01^{\circ} \mathrm{C}$ and an exothermic peak at $419^{\circ} \mathrm{C}$ appeared. Blank nanoparticles of $\mathrm{Chi}$ and $\mathrm{Chi}-\mathrm{PF}$ physically mixed with GC (Figure $6 \mathrm{C}$ and $\mathrm{E}$, respectively) show similar endothermic peaks at the same temperature. In both cases, no melting peak was observed when GC-loaded Chi nanoparticles (Figure 6A) and GC-loaded Chi-PF nanoparticles (Figure 6G) were studied. It can be concluded that the drug is incorporated in its amorphous or disordered crystalline phase inside the nanoparticle matrix.

\section{In vitro release profile}

We used different formulations containing PF (10\%, 15\%, and $20 \% \mathrm{w} / \mathrm{w}$ ) to investigate the drug-release behavior of GC-loaded Chi-PF nanoparticles. The results were compared with GC-loaded Chi nanoparticles. The percentage of cumulative release was $85.86 \%, 79.58 \%, 71.23 \%$, and $57.74 \%$ for Chi and Chi-PF nanoparticles incorporated with $10 \%, 15 \%$, and $20 \% \mathrm{w} / \mathrm{w}$ PF after 72 hours, respectively. All formulations show an initial drug-release burst up to 6 hours continued by a steady-state release. The release medium easily penetrates into the tortuous paths on the peripheral surface of the nanoparticle matrix and dissolves the drug. The dissolved drug rapidly diffuses into the release medium near the surface of the nanoparticles, and exhibits a rapid burst release. Although our intention was to achieve a controlled

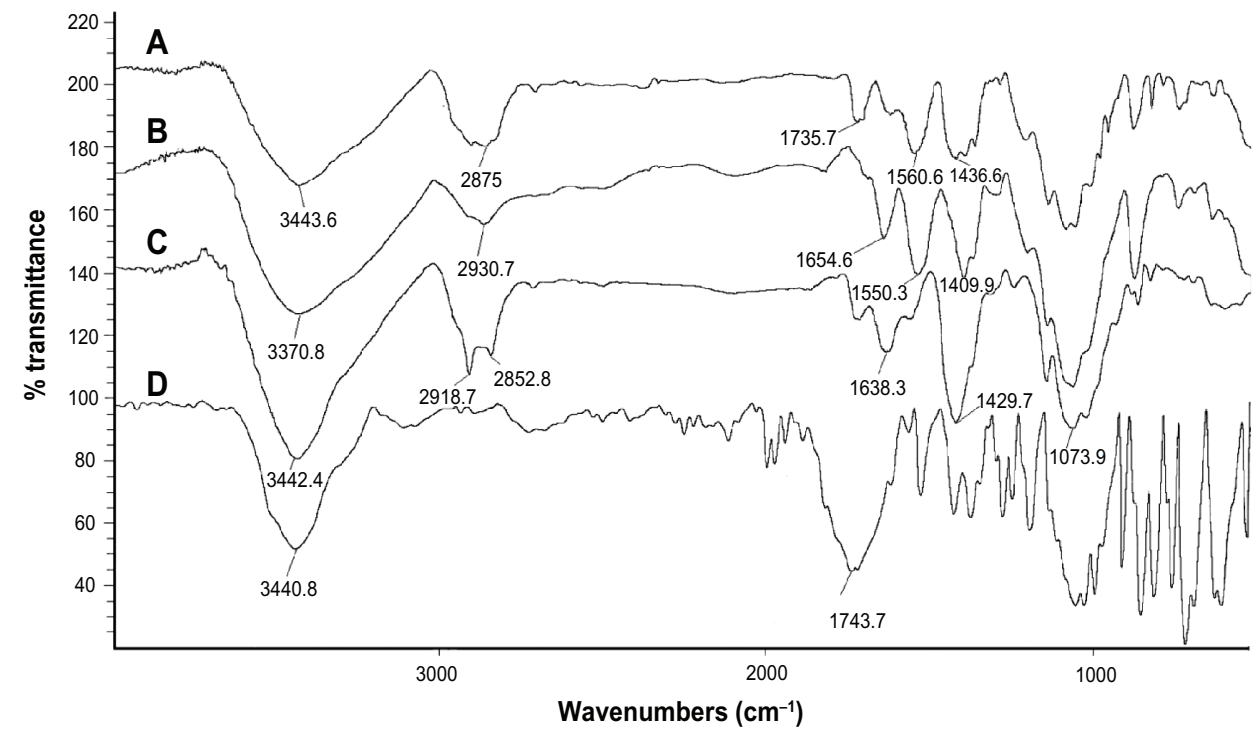

Figure 4 Fourier transform infrared spectra of (A) GC-loaded Chi nanoparticles, (B) blank Chi nanoparticles, (C) Chi alone, and (D) GC. Abbreviations: Chi, chitosan; GC, gemcitabine; PF, Pluronic $F^{\circledR} 127$. 


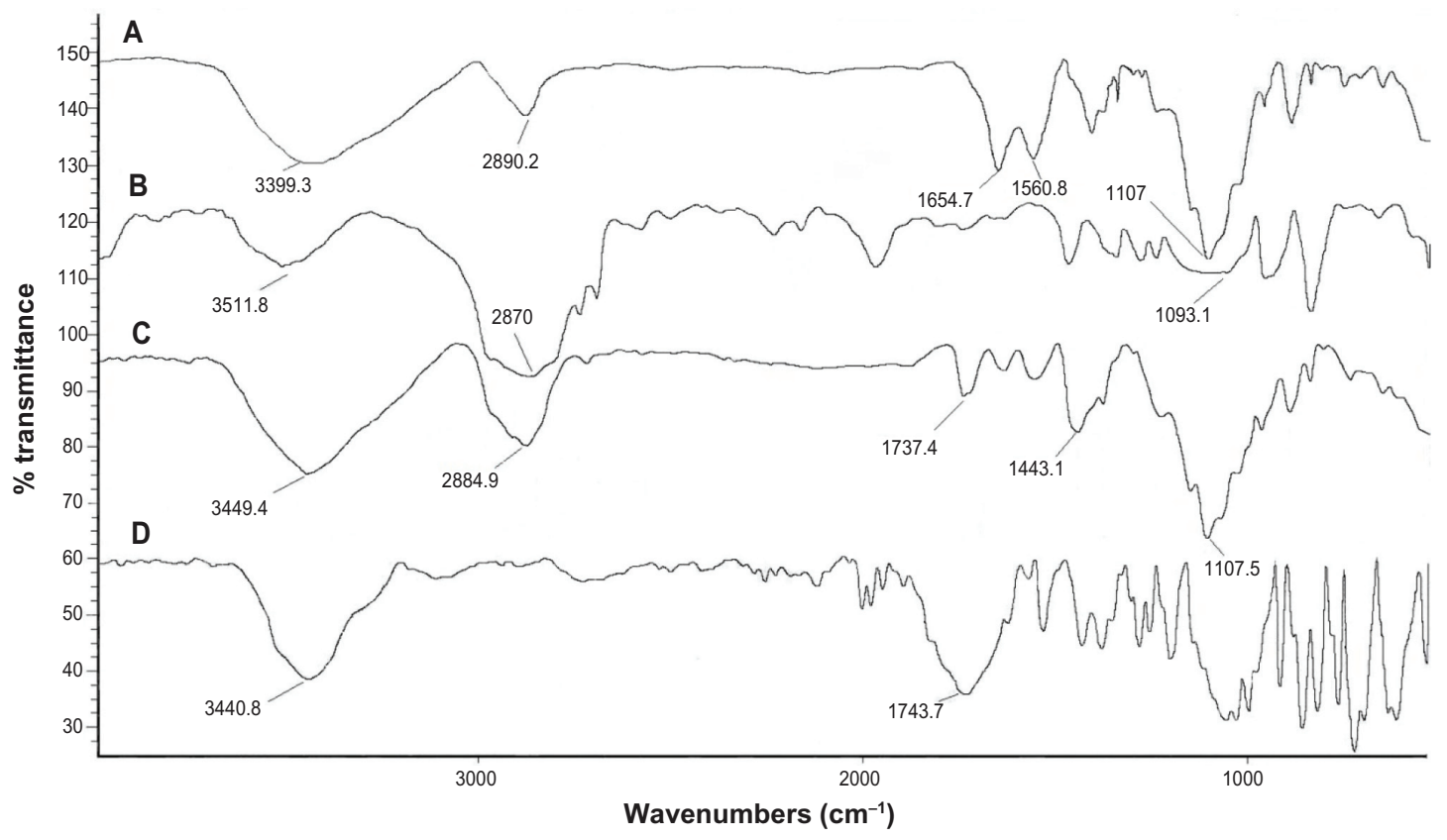

Figure 5 Fourier transform infrared spectra of (A) blank Chi-PF nanoparticles, (B) PF, (C) GC-loaded Chi-PF nanoparticles, and (D) GC.

Abbreviations: Chi, chitosan; GC, gemcitabine; PF, Pluronic $\mathrm{F}^{\circledR} / 27$.

release profile by $\mathrm{PF}$ augmentation (Figure 7), incorporation of PF led to a lower release rate for Chi-PF nanoparticles in comparison with Chi nanoparticles. This might be due to the presence of $\mathrm{PF}$, which has inhibited some of the paths for drug release. In addition, studies observed that PF solutions show a thermoreversible gelation property at concentrations higher than $15 \%-20 \% \mathrm{w} / \mathrm{w}$. This copolymer is liquid at $4^{\circ} \mathrm{C}-8^{\circ} \mathrm{C}$. At body temperature, this polymer aggregates into micelles forming a semisolid gel with a dehydrated core of PPO surrounded by hydrated PEO. This micellar nature prolongs the

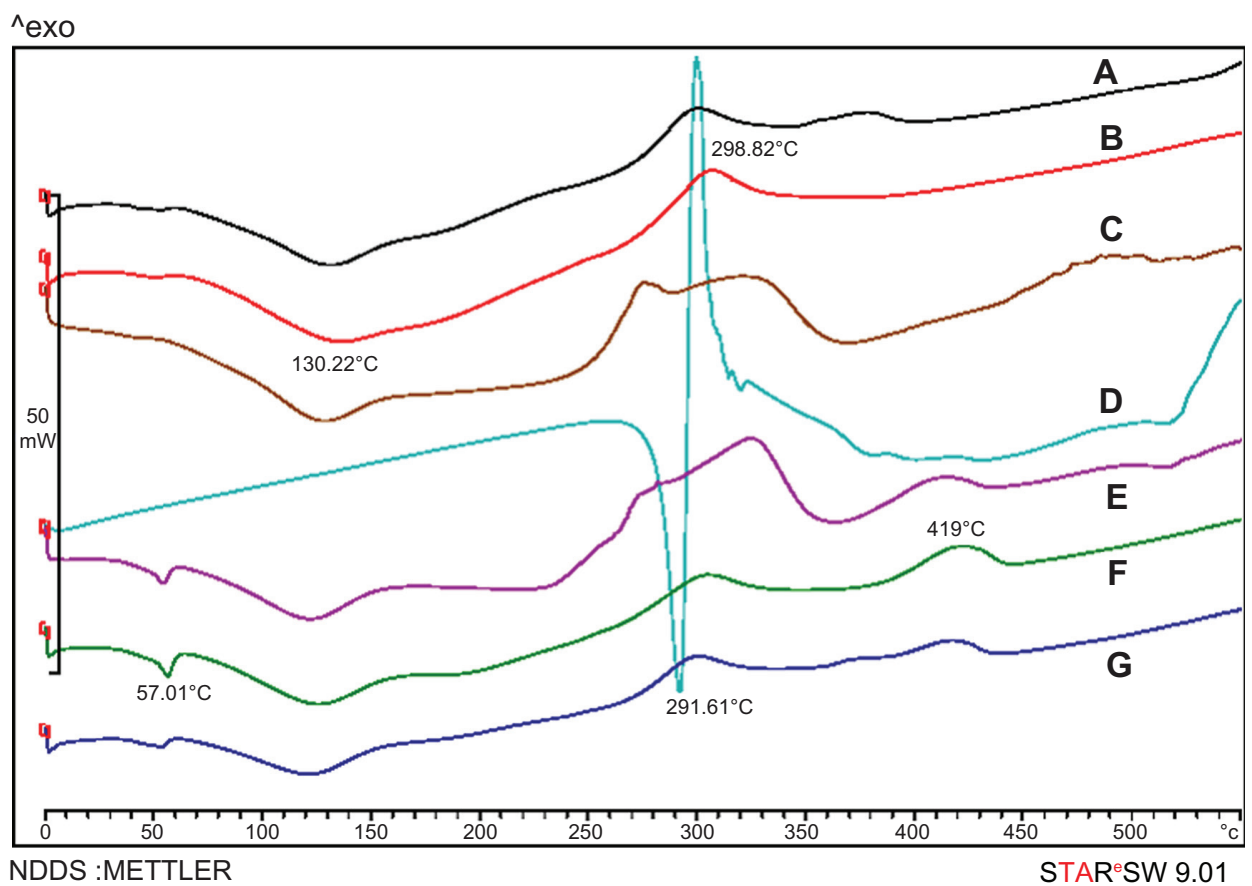

Figure 6 Differential scanning calorimetry thermograms of (A) GC-loaded Chi nanoparticles, (B) blank Chi nanoparticles, (C) physical mixture of GC and blank Chi nanoparticles, (D) GC, (E) physical mixture of GC and Chi-PF nanoparticles, (F) blank Chi-PF nanoparticles, and (G) GC-loaded Chi-PF nanoparticles. Abbreviations: Chi, chitosan; GC, gemcitabine; PF, Pluronic $\mathrm{F}^{\circledR} \mathrm{I} 27$. 


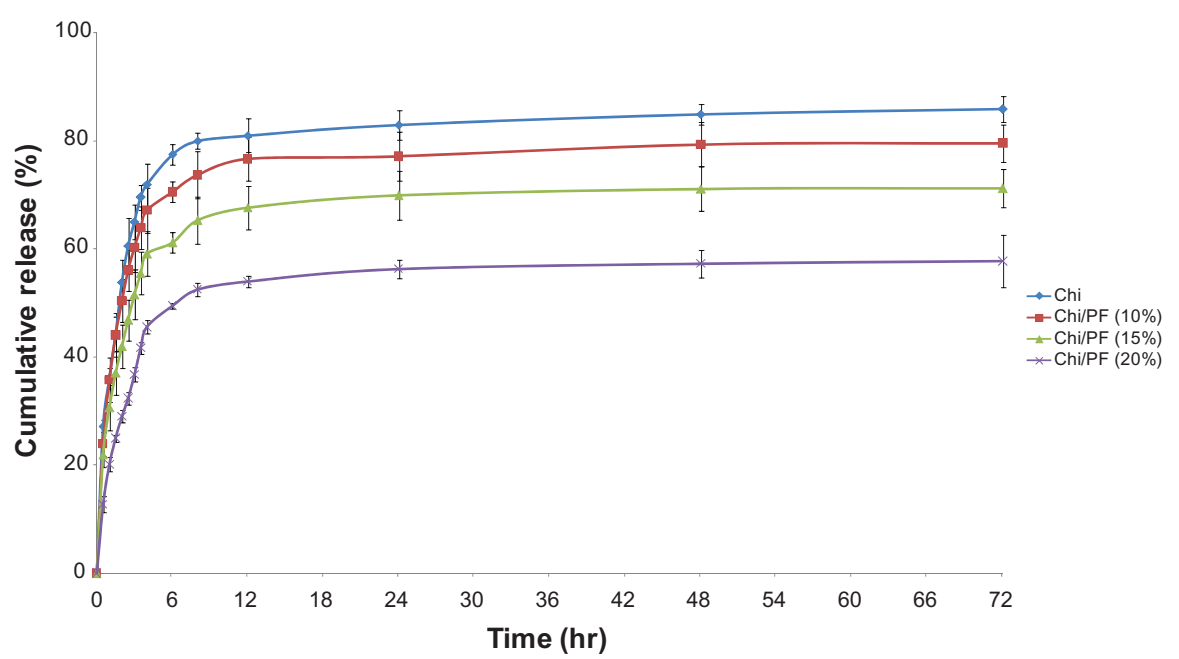

Figure 7 In vitro release of gemcitabine from gemcitabine-loaded $\mathrm{Chi}$ and different formulations of Chi-PF nanoparticles in $\mathrm{PBS}(\mathrm{pH}=7.4)$ at $37^{\circ} \mathrm{C}(\mathrm{n}=3)$. Abbreviations: Chi, chitosan; PBS, phosphate-buffered saline; PF, Pluronic F® 127.

release rate of GC. As discussed previously, the higher the PF concentration, the more rigid gel forms, therefore leading to a lower release rate. ${ }^{46}$

\section{Mucoadhesion study}

The mucoadhesive behavior of Chi and Chi-PF nanoparticles were studied through mucus glycoprotein assay. Results are reported as mucoadhesion percentages in Figure 8; both formulations show high mucoadhesion in comparison with negative control. Incorporation of PF did not show a significant change in mucoadhesiveness. High mucoadhesivity of the nanoparticles is attributed to the hydrogen bond and ionic interaction of the positive charge of Chi amino groups with mucin chains. ${ }^{47}$ Aghaei Moghaddam et al reported higher mucoadhesion for smaller particles than for larger particles. ${ }^{33}$ The small nanoparticle size provides a large surface

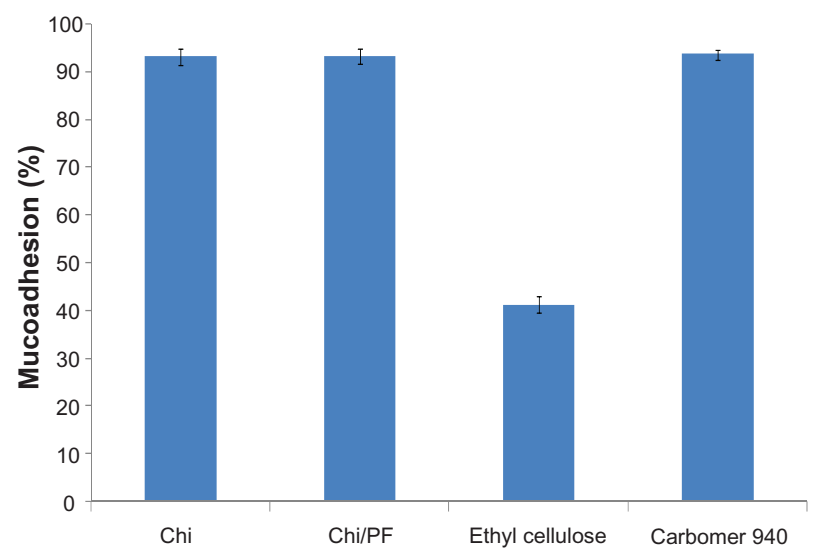

Figure 8 Mucoadhesion (\%) of Chi and Chi-PF nanoparticles (PF = 20\% w/w) in comparison with controls $(n=3)$.

Abbreviations: Chi, chitosan; PF, Pluronic $F^{\circledR} / 27$. area and increase in mucin adsorption, which leads to a high mucoadhesive property for the nanoparticles.

\section{Cytotoxicity assay}

The cytotoxicity of blank and GC-loaded Chi and Chi-PF nanoparticles and drug alone was assessed by MTT assay on the HT-29 colon carcinoma cell line. Blank nanoparticles and drug alone have no toxic effect on applied cells, but this effect was observed for GC-loaded nanoparticles (Figures 9A and 9B). There was no noticeable difference between the cytotoxic effect of GC-loaded nanoparticles prepared with Chi alone and Chi-PF (half-maximal concentration against $18.10 \mu \mathrm{M}$ and $17.47 \mu \mathrm{M}$, respectively). Therefore, it can be concluded that incorporation of PF does not influence the cytotoxicity of the nanoparticles. GC is a hydrophilic anticancer drug that needs membrane transporters to enter the cells. ${ }^{29}$ Cells can uptake nanoparticles by endocytosis ${ }^{48}$ mediated by a clathrin-mediated process. ${ }^{49}$ Therefore nanoparticles can act as drug delivery systems that facilitate drug entrance into the cells. HT-29 is a mucinproducing cell line. ${ }^{50,51}$ As discussed previously, Chi and Chi-PF nanoparticles showed highly mucoadhesive properties, therefore GC-loaded Chi nanoparticles can interact with the mucin produced by HT-29 cell line and can become localized on the cell surface. Other studies have reported improved uptake of mucoadhesive Chi nanoparticles by the HT-29 cell line. ${ }^{52}$ The release of GC from the adhered nanoparticles increases the availability of the drug for membrane transport and increases the accumulation of the drug inside the cells. ${ }^{28}$ Both mechanisms described could be responsible for increased cytotoxicity of GC-loaded nanoparticles. 

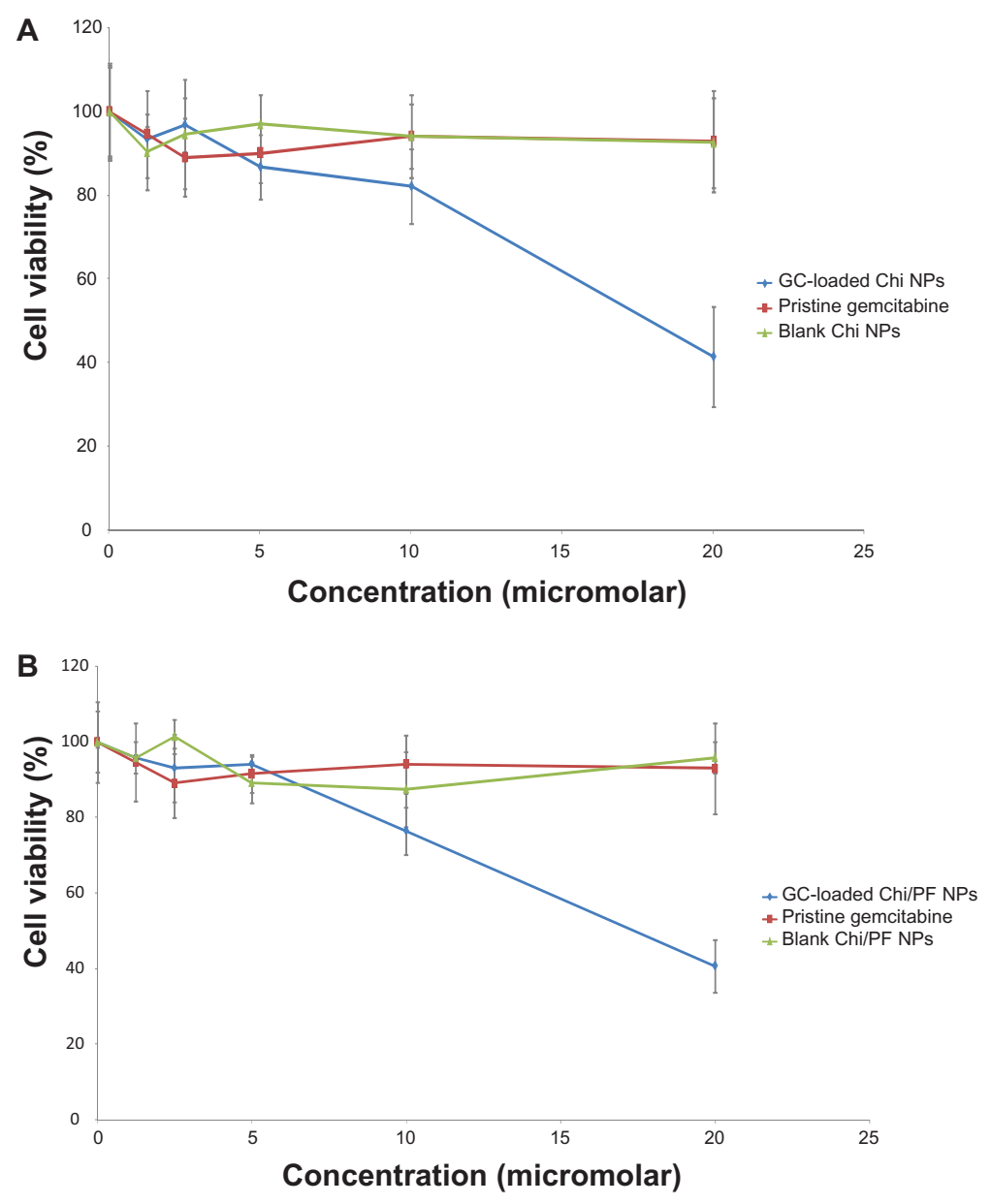

Figure 9 The effect of GC-loaded Chi (A) and Chi-PF (B) nanoparticles on the viability of HT-29 $(n=4)$.

Abbreviations: Chi, chitosan; GC, gemcitabine; PF, Pluronic $\mathrm{F}^{\circledR} / 27$.

\section{Conclusion}

GC-loaded nanoparticles were prepared by a mild process without using organic solvents. A particle size of less than $200 \mathrm{~nm}$ and high encapsulation efficiency demonstrated good mucoadhesion properties. GC-loaded nanoparticles could be considered as a good candidate for oral delivery of the anticancer drug. This hypothesis is partially proven by a cell culture study on the HT-29 colon carcinoma cell line. However other studies should be performed to improve the efficacy of these nanoparticles.

\section{Disclosure}

The authors report no conflicts of interest in this work.

\section{References}

1. Suri S, Fenniri H, Singh B. Nanotechnology-based drug delivery systems. J Occup Med Toxicol. 2007;2(1):16.

2. Wang X, Yang L, Chen ZG, Shin DM. Application of nanotechnology in cancer therapy and imaging. CA Cancer J Clin. 2008;58(2): $97-110$.

3. Minchinton AI, Tannock IF. Drug penetration in solid tumours. Nat Rev Cancer. 2006;6(8):583-592.
4. Yousefpour P,Atyabi F, Vasheghani Farahani E, Sakhtianchi R, Dinarvand R Polyanionic carbohydrate doxorubicin-dextran nanocomplex as a delivery system for anticancer drugs: in vitro analysis and evaluations. Int J Nanomedicine. 2011;6:1487-1496.

5. Ferrari M. Cancer nanotechnology: opportunities and challenges. Nat Rev Cancer. 2005;5(3):161-171.

6. Cuenca AG, Jiang H, Hochwald SN, et al. Emerging implications of nanotechnology on cancer diagnostics and therapeutics. Cancer. 2006;107(3):459-466.

7. Agnihotri SA, Mallikarjuna NN, Aminabhavi TM. Recent advances on chitosan-based micro-and nanoparticles in drug delivery. J Control Release. 2004;100(1):5-28.

8. Calvo P, Remuñán López C, Vila Jato J, Alonso M. Novel hydrophilic chitosan polyethylene oxide nanoparticles as protein carriers. $J$ Appl Polym Sci. 1997;63(1):125-132.

9. Shah S, Pal A, Kaushik V, Devi S. Preparation and characterization of venlafaxine hydrochloride loaded chitosan nanoparticles and in vitro release of drug. J Appl Polym Sci. 2009;112(5):2876-2887.

10. Atyabi F, Talaie F, Dinarvand R. Thiolated chitosan nanoparticles as an oral delivery system for amikacin: in vitro and ex vivo evaluations. J Nanosci Nanotechnol. 2009;9(8):4593-4603.

11. Saremi S, Atyabi F, Akhlaghi SP, Ostad SN, Dinarvand R. Thiolated chitosan nanoparticles for enhancing oral absorption of docetaxel: preparation, in vitro and ex vivo evaluation. Int J Nanomedicine. 2011;6:119-128.

12. Pan Y, Li Y, Zhao H, et al. Bioadhesive polysaccharide in protein delivery system: chitosan nanoparticles improve the intestinal absorption of insulin in vivo. Int J Pharm. 2002;249(1-2):139-147. 
13. Talasaz AHH, Ghahremankhani AA, Moghadam SH, et al. In situ gel forming systems of poloxamer 407 and hydroxypropyl cellulose or hydroxypropyl methyl cellulose mixtures for controlled delivery of vancomycin. J Appl Polym Sci. 2008;109(4):2369-2374.

14. Taheri A, Atyabi F, Dinarvnd R. Temperature-responsive and biodegradable PVA: PVP k30: poloxamer 407 hydrogel for controlled delivery of human growth hormone (hGH). J Pediatr Endocrinol Metab. 2011;24(3-4):175-179.

15. Mortensen K. Block copolymer in aqueous solution: Micelle formation and hard-sphere crystallization. Trends in Colloid and Interface Science VII. 1993:72-75.

16. Zhou Z, Chu B. Light-scattering study on the association behavior of triblock polymers of ethylene oxide and propylene oxide in aqueous solution. J Colloid Interf Sci. 1988;126(1):171-180.

17. Wanka G, Hoffmann H, Ulbricht W. The aggregation behavior of poly(oxyethylene)-poly-(oxypropylene)-poly-(oxyethylene)-block-copolymers in aqueous solution. Colloid Polym Sci. 1990;268(2):101-117.

18. Bohorquez M, Koch C, Trygstad T, Pandit N. A study of the temperature-dependent micellization of Pluronic F127. J Colloid Interf Sci. 1999;216(1):34-40.

19. Cabana A, Aït-Kadi A, Juhász J. Study of the gelation process of polyethylene oxidea-polypropylene oxideb-polyethylene oxideacopolymer (poloxamer 407) aqueous solutions. J Colloid Interf Sci. 1997;190(2):307-312.

20. Wenzel JGW, Balaji K, Koushik K, et al. Pluronic ${ }^{\circledR}$ F127 gel formulations of Deslorelin and GnRH reduce drug degradation and sustain drug release and effect in cattle. J Control Release. 2002;85(1-3):51-59.

21. Veyries M, Couarraze G, Geiger S, et al. Controlled release of vancomycin from poloxamer 407 gels. Int J Pharm. 1999;192(2):183-193.

22. Reddy LH, Dubernet C, Mouelhi SL, et al. A new nanomedicine of gemcitabine displays enhanced anticancer activity in sensitive and resistant leukemia types. J Control Release. 2007;124(1-2):20-27.

23. Immordino ML, Brusa P, Rocco F, et al. Preparation, characterization, cytotoxicity and pharmacokinetics of liposomes containing lipophilic gemcitabine prodrugs. J Control Release. 2004;100(3):331-346.

24. Grinberg O, Gedanken A, Patra CR, et al. Sonochemically prepared BSA microspheres containing Gemcitabine, and their potential application in renal cancer therapeutics. Acta Biomater. 2009;5(8):3031-3037.

25. Mackey JR, Mani RS, Selner M, et al. Functional nucleoside transporters are required for gemcitabine influx and manifestation of toxicity in cancer cell lines. Cancer Res. 1998;58(19):4349.

26. Rauchwerger DR, Firby PS, Hedley DW, Moore MJ. Equilibrativesensitive nucleoside transporter and its role in gemcitabine sensitivity. Cancer Res. 2000;60(21):6075.

27. Reddy LH, Couvreur P. Novel approaches to deliver gemcitabine to cancers. Curr Pharm Des. 2008;14(11):1124-1137.

28. Trickler WJ, Khurana J, Nagvekar AA, Dash AK. Chitosan and glyceryl monooleate nanostructures containing gemcitabine: potential delivery system for pancreatic cancer treatment. AAPS PharmSciTech. 2010;11(1):392-401.

29. Arias JL, Reddy LH, Couvreur P. Polymeric nanoparticulate system augmented the anticancer therapeutic efficacy of gemcitabine. J of Drug Target. 2009;17(8):586-598.

30. Wang CX, Huang LS, Hou LB, et al. Antitumor effects of polysorbate- 80 coated gemcitabine polybutylcyanoacrylate nanoparticles in vitro and its pharmacodynamics in vivo on C6 glioma cells of a brain tumor model. Brain research. 2009;1261:91-99.

31. Patra CR, Bhattacharya R, Wang E, et al. Targeted delivery of gemcitabine to pancreatic adenocarcinoma using cetuximab as a targeting agent. Cancer Res. 2008;68(6):1970-1978.

32. Gang J, Park SB, Hyung W, et al. Magnetic poly epsilon-caprolactone nanoparticles containing $\mathrm{Fe} 3 \mathrm{O} 4$ and gemcitabine enhance anti-tumor effect in pancreatic cancer xenograft mouse model. $J$ Drug Target. 2007;15(6):445-453.
33. Moghaddam FA, Atyabi F, Dinarvand R. Preparation and in vitro evaluation of mucoadhesion and permeation enhancement of thiolated chitosan-pHEMA core-shell nanoparticles. Nanomedicine. 2009;5(2):208-215.

34. Akhlaghi SP, Saremi S, Ostad SN, Dinarvand R, Atyabi F. Discriminated effects of thiolated chitosan-coated pMMA paclitaxelloaded nanoparticles on different normal and cancer cell lines. Nanomedicine. 2010;6(5):689-697.

35. Papadimitriou S, Bikiaris D, Avgoustakis K, Karavas E, Georgarakis M. Chitosan nanoparticles loaded with dorzolamide and pramipexole. Carbohyd Polym. 2008;73(1):44-54.

36. He P, Davis SS, Illum L. In vitro evaluation of the mucoadhesive properties of chitosan microspheres. Int J Pharm. 1998;166(1): $75-88$.

37. MacLaughlin FC, Mumper RJ, Wang J, et al. Chitosan and depolymerized chitosan oligomers as condensing carriers for in vivo plasmid delivery. J Control Release. 1998;56(1-3):259-272.

38. Ajun W, Yan S, Li G, Huili L. Preparation of aspirin and probucol in combination loaded chitosan nanoparticles and in vitro release study. Carbohyd Polym. 2009;75(4):566-574.

39. Katas H, Alpar HO. Development and characterisation of chitosan nanoparticles for siRNA delivery. $J$ Control Release. 2006;115(2):216-225.

40. Zheng Y, Wu Y, Yang W, et al. Preparation, characterization, and drug release in vitro of chitosan glycyrrhetic acid nanoparticles. J Pharm Sci. 2006;95(1):181-191.

41. Gan Q, Wang T. Chitosan nanoparticle as protein delivery carrier systematic examination of fabrication conditions for efficient loading and release. Colloids Surf B Biointerf. 2007;59(1):24-34.

42. Ajun W, Yan S, Li G, Huili L. Preparation of aspirin and probucol in combination loaded chitosan nanoparticles and in vitro release study. Carbohyd Polym. 2009;75(4):566-574.

43. Rokhade AP, Shelke NB, Patil SA, Aminabhavi TM. Novel hydrogel microspheres of chitosan and pluronic F-127 for controlled release of 5-fluorouracil. J Microencapsul. 2007;24(3):274-288.

44. Cho CW, Shin S, Oh IJ. Thermorheologic properties of aqueous solutions and gels of poloxamer 407. Drug Dev Ind Pharm. 1997;23(12):1227-1232.

45. Wu Y, Yang W, Wang C, Hu J, Fu S. Chitosan nanoparticles as a novel delivery system for ammonium glycyrrhizinate. Int $J$ Pharm. 2005;295(1-2):235-245.

46. Rokhade AP, Shelke NB, Patil SA, Aminabhavi TM. Novel hydrogel microspheres of chitosan and pluronic F-127 for controlled release of 5-fluorouracil. J Microencapsul. 2007;24(3):274-288.

47. Bravo-Osuna I, Vauthier C, Farabollini A, Palmieri GF, Ponchel G. Mucoadhesion mechanism of chitosan and thiolated chitosan-poly (isobutyl cyanoacrylate) core-shell nanoparticles. Biomaterials. 2007;28(13):2233-2243.

48. Davda J, Labhasetwar V. Characterization of nanoparticle uptake by endothelial cells. Int J Pharm. 2002;233(1-2):51-59.

49. Huang M, Ma Z, Khor E, Lim LY. Uptake of FITC-chitosan nanoparticles by A549 cells. Pharm Res. 2002;19(10):1488-1494.

50. Shekels LL, Lyftogt CT, Ho SB. Bile acid-induced alterations of mucin production in differentiated human colon cancer cell lines* 1 . Int J Biochem Cell Biol. 1996;28(2):193-201.

51. Niv Y, Byrd JC, Ho SB, Dahiya R, Kim YS. Mucin synthesis and secretion in relation to spontaneous differentiation of colon cancer cells in vitro. Int J Cancer. 1992;50(1):147-152.

52. Prego C, Fabre M, Torres D, Alonso M. Efficacy and mechanism of action of chitosan nanocapsules for oral peptide delivery. Pharm Res. 2006;23(3):549-556. 
International Journal of Nanomedicine

Dovepress

\section{Publish your work in this journal}

The International Journal of Nanomedicine is an international, peerreviewed journal focusing on the application of nanotechnology in diagnostics, therapeutics, and drug delivery systems throughou the biomedical field. This journal is indexed on PubMed Central, MedLine, CAS, SciSearch ${ }^{\circledR}$, Current Contents ${ }^{\circledR} /$ Clinical Medicine,
Journal Citation Reports/Science Edition, EMBase, Scopus and the Elsevier Bibliographic databases. The manuscript management system is completely online and includes a very quick and fair peer-review system, which is all easy to use. Visit http://www.dovepress.com/ testimonials.php to read real quotes from published authors.

Submit your manuscript here: http://www.dovepress.com/international-journal-of-nanomedicine-journal 